\title{
Dynamic torque calibration by means of model parameter identification
}

\author{
Leonard Klaus ${ }^{1}$, Barbora Arendacká ${ }^{2}$, Michael Kobusch $^{1}$, Thomas Bruns ${ }^{1}$ \\ ${ }^{1}$ Physikalisch-Technische Bundesanstalt (PTB), Bundesallee 100, 38116 Braunschweig, Germany \\ ${ }^{2}$ Physikalisch-Technische Bundesanstalt (PTB), Abbestraße 2-12, 10587 Berlin, Germany
}

\section{ABSTRACT}

For the dynamic calibration of torque transducers, a model of the unmounted transducer and an extended model of the mounted transducer including the measuring device have been developed. The dynamic behaviour of a torque transducer under test will be described by its model parameters. This paper presents the models comprising the known parameters of the measuring device and the unknown parameters of the transducer and how the calibration measurements are going to be carried out. The principle for the identification of the transducer's model parameters from measurement data is described using a least squares approach. The influence of a variation of the transducer's parameters on the frequency response of the expanded model is analysed.

\section{Section: RESEARCH PAPER}

Keywords: model parameter identification; dynamic torque calibration; dynamic measurement; mechanical model

Citation: Leonard Klaus, Barbora Arendacká, Michael Kobusch, Thomas Bruns, Dynamic torque calibration by means of model parameter identification, Acta IMEKO, vol. 4, no. 2, article 7, June 2015, identifier: IMEKO-ACTA-04 (2015)-02-07

Editor: Paolo Carbone, University of Perugia, Italy

Received September 24, 2014; In final form December 11, 2014; Published June 2015

Copyright: @ 2015 IMEKO. This is an open-access article distributed under the terms of the Creative Commons Attribution 3.0 License, which permits unrestricted use, distribution, and reproduction in any medium, provided the original author and source are credited

Funding: This work is part of the European Metrology Research Programme (EMRP) Joint Research Project IND09 - "Traceable Dynamic Measurement of Mechanical Quantities". The EMRP is jointly funded by the EMRP participating countries within EURAMET and the European Union.

Corresponding author: Leonard Klaus, Email: leonard.klaus@ptb.de

\section{INTRODUCTION}

Research in the field of dynamic calibration of torque transducers has been carried out in the context of the European Metrology Research Programme (EMRP) Joint Research Project IND09 "Traceable Dynamic Measurement of Mechanical Quantities" [1], [2]. An existing prototype measuring device [3] was modernised and extended, and a model-based description of the dynamic behaviour of torque transducers was developed [4]. The model of the transducer will be used to describe its dynamic behaviour in a later industrial application. The modelling is necessary, because torque transducers are always coupled on both ends to a given mechanical environment, which may influence the transducer's dynamic behaviour. In case of the calibration this environment differs from that of a subsequent use in industry.

For future dynamic torque calibrations, it will be necessary to identify the model parameters of a transducer to be calibrated from measurement data.
This contribution is an extended version of a contribution to the IMEKO International TC3, TC5 and TC22 Conference 2014 [5].

\section{MODEL}

The mathematical model used to represent the torque transducer assumes a linear and time invariant (LTI) system which consists of two mass moment of inertia (MMOI) elements connected by a torsional spring and a damper in parallel.

To be able to include the previously mentioned influence of the mechanical environment in the model-based description of the dynamic behaviour of the transducer, it was necessary to extend the model of the transducer to consider the mounted transducer including the dynamic torque measuring device (i.e. the mechanical environment in case of a calibration).

This extended model represents the physical components of the measuring device and the transducer under test while assuming LTI behaviour (see Figure 1). It consists of elements for the mass moment of inertia, the torsional spring and the 


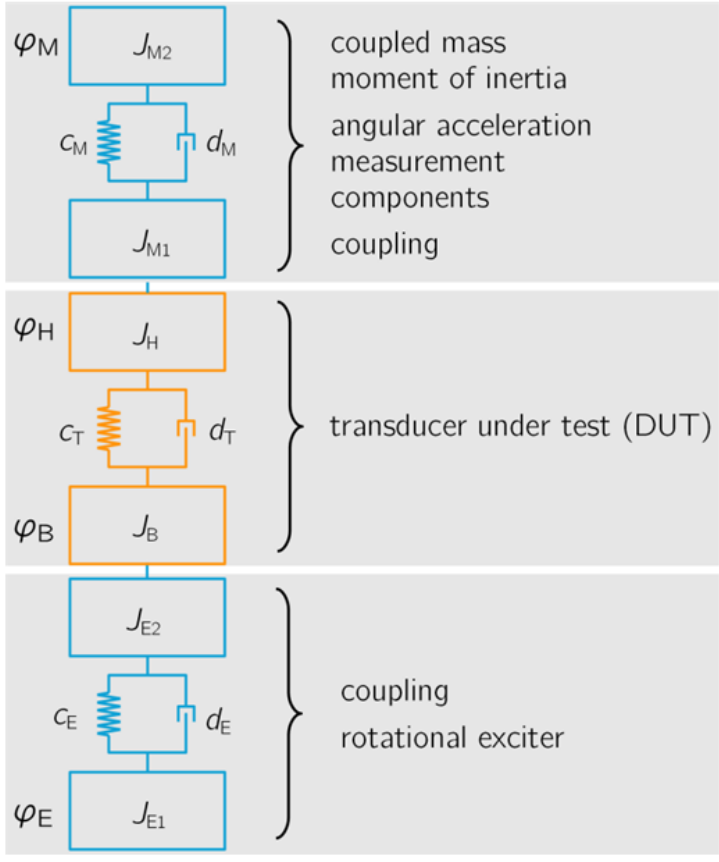

Figure 1. Model of the dynamic torque calibration device (marked in blue) including the transducer under test (marked in orange).

torsional damper. The equation of motion is described as an inhomogeneous system of ordinary differential equations:

$$
\boldsymbol{J} \ddot{\boldsymbol{\varphi}}+\boldsymbol{D} \dot{\boldsymbol{\varphi}}+\boldsymbol{C} \boldsymbol{\varphi}=\boldsymbol{M}
$$

In this equation $\boldsymbol{J}$ denotes the mass moment of inertia matrix, $\boldsymbol{D}$ the damping matrix, $\boldsymbol{C}$ the stiffness matrix and $\boldsymbol{\varphi}$ the angle vector and its derivative vectors $(\ddot{\boldsymbol{\varphi}}, \dot{\boldsymbol{\varphi}})$, respectively. The forced excitation is described by $\boldsymbol{M}$.

For the described model as depicted in Figure 1, the model approach leads to the mass moment of inertia matrix

$$
\boldsymbol{J}=\left[\begin{array}{cccc}
J_{\mathrm{M} 2} & 0 & 0 & 0 \\
0 & \left(J_{\mathrm{M} 1}+J_{\mathrm{H}}\right) & 0 & 0 \\
0 & 0 & \left(J_{\mathrm{B}}+J_{\mathrm{E} 2}\right) & 0 \\
0 & 0 & 0 & J_{\mathrm{E} 1}
\end{array}\right]
$$

the damping matrix

$$
\boldsymbol{D}=\left[\begin{array}{cccc}
d_{\mathrm{M}} & -d_{\mathrm{M}} & 0 & 0 \\
-d_{\mathrm{M}} & \left(d_{\mathrm{M}}+d_{\mathrm{T}}\right) & -d_{\mathrm{T}} & 0 \\
0 & -d_{\mathrm{T}} & \left(d_{\mathrm{T}}+d_{\mathrm{E}}\right) & -d_{\mathrm{E}} \\
0 & 0 & -d_{\mathrm{E}} & d_{\mathrm{E}}
\end{array}\right]
$$

and the corresponding stiffness matrix

$$
\boldsymbol{C}=\left[\begin{array}{cccc}
c_{M} & -c_{M} & 0 & 0 \\
-c_{M} & \left(c_{M}+c_{T}\right) & -c_{T} & 0 \\
0 & -c_{T} & \left(c_{T}+c_{E}\right) & -c_{E} \\
0 & 0 & -c_{E} & c_{E}
\end{array}\right]
$$

The angle vector and its derivative vectors $\boldsymbol{\varphi}, \dot{\boldsymbol{\varphi}}, \ddot{\boldsymbol{\varphi}}$ are given by

$$
\boldsymbol{\varphi}=\left[\begin{array}{c}
\varphi_{\mathrm{M}} \\
\varphi_{\mathrm{H}} \\
\varphi_{\mathrm{B}} \\
\varphi_{\mathrm{E}}
\end{array}\right] \quad \dot{\boldsymbol{\varphi}}=\left[\begin{array}{c}
\dot{\varphi}_{\mathrm{M}} \\
\dot{\varphi}_{\mathrm{H}} \\
\dot{\varphi}_{\mathrm{B}} \\
\dot{\varphi}_{\mathrm{E}}
\end{array}\right] \quad \ddot{\boldsymbol{\varphi}}=\left[\begin{array}{c}
\ddot{\varphi}_{\mathrm{M}} \\
\ddot{\varphi}_{\mathrm{H}} \\
\ddot{\varphi}_{\mathrm{B}} \\
\ddot{\varphi}_{\mathrm{E}}
\end{array}\right]
$$

The forced excitation of the rotational exciter is given by

$\boldsymbol{M}=\left[\begin{array}{c}0 \\ 0 \\ 0 \\ M\end{array}\right]$
Table 1: Model parameters of the measuring device and of the device under test.

\begin{tabular}{l|cc} 
& $\begin{array}{c}\text { Known parameters of } \\
\text { the measuring device }\end{array}$ & $\begin{array}{c}\text { Unknown parameters } \\
\text { of the DUT }\end{array}$ \\
\hline MMOI & $J_{\mathrm{M} 2}, J_{\mathrm{M} 1}, J_{\mathrm{E} 2}$ & $J_{\mathrm{H}}, J_{\mathrm{B}}$ \\
Torsional stiffness & $c_{\mathrm{M}}, c_{\mathrm{E}}$ & $c_{\mathrm{T}}$ \\
Damping & $d_{\mathrm{M}}, d_{\mathrm{E}}$ & $d_{\mathrm{T}}$
\end{tabular}

\section{KNOWN AND UNKNOWN MODEL PARAMETERS}

To be able to identify the unknown model parameters of the torque transducer, it was necessary to identify the model parameters of the measuring device first. To this end, dedicated auxiliary measuring set-ups for the determination of the mass moment of inertia, torsional stiffness [4] and torsional damping [6] were developed. Based on the measurement results from these set-ups, the previously unknown model parameters of the dynamic torque calibration device have been determined.

A similar determination of the model parameters of the transducer under test is not possible. Due to the unknown mechanical design and the lack of knowledge about the actual dynamic behaviour, a dynamic calibration remains necessary.

The extended model of the measuring device now consists of a set of known model parameters, which represents the components of the measuring device, and of a set of unknown model parameters, representing the transducer under test (see Table 1), which need to be identified.

\section{DATA ACQUISITION AND ANALYSIS}

For the calibration measurement with a transducer under test, a sinusoidal excitation with given frequencies is generated by a rotational exciter. The frequency response function of the drive shaft of the measuring device depends on the device under test (see Figure 2). The control of excitation frequency and vibration magnitude, including abort conditions and a predetermination of the frequency response of each set-up, is carried out by means of a closed-loop vibration controller.

The excitation frequencies are based on the recommendations from [7]. They are equally spaced in logarithmic scale in the frequency domain. The $1 / 3$ octave series was chosen for low and high frequencies far from the resonance frequency, and the narrower spaced 1/12 octave series was chosen for frequencies close to the resonance frequency, respectively. The frequency range of excitation ranges from $10 \mathrm{~Hz}$ up to $1 \mathrm{kHz}$.

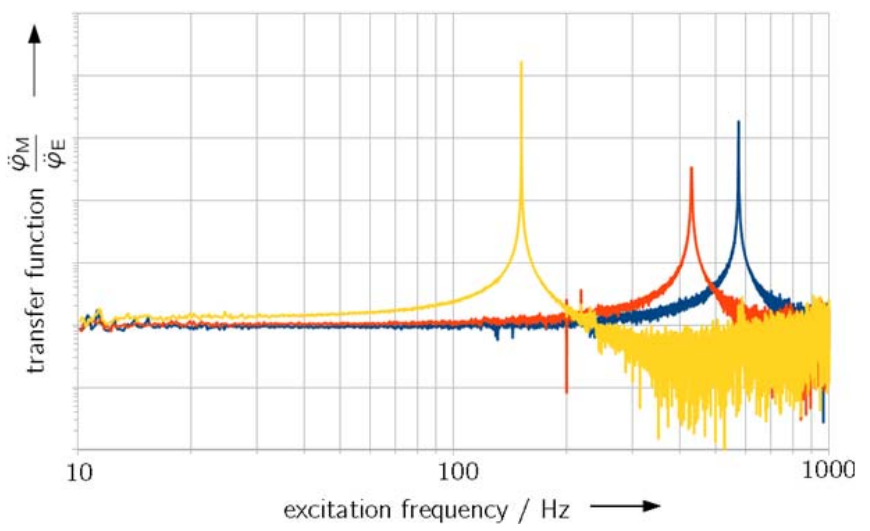

Figure 2. Frequency response of the measuring device with three DUTs of different torsional stiffness and $\mathrm{MMOI}$ measured with random noise excitation. 
The angle of excitation at the top $\varphi_{\mathrm{M}}(t)$ is measured by means of a laser Doppler vibrometer for rotational oscillations. The rotational acceleration at the bottom $\ddot{\varphi}_{\mathrm{E}}(t)$ is measured by means of an integrated angular accelerometer. For calibration measurements, these two quantities and the output of the transducer $u_{\text {DUT }}(t)$ are acquired with a four-channel data acquisition system. Two high speed sampling inputs acquire the raw signal of the interferometer with a high sampling rate; two high precision inputs acquire the voltage outputs of the angular accelerometer and of the transducer under test. The frequency modulated interferometer output signal is demodulated by software and down-sampled to the lower sampling rate of the high precision input channels afterwards.

The three input quantities are monofrequent sinusoids with the angular frequency $\omega=2 \pi f$, the phase offset $\theta$ and the magnitude $A$ as follows

$$
y(t)=A \cdot \sin (\omega \cdot t+\theta)
$$

Such a signal can be described by sine and cosine components as follows

$$
y(t)=a \cdot \cos (\omega \cdot t)+b \cdot \sin (\omega \cdot t)
$$

The parameters $A$ and $\varphi$ of Eq. 3 can be derived from $a$ and $b$ of Eq. 4 as

$$
A=\sqrt{a^{2}+b^{2}}, \quad \varphi=\arctan \left(\frac{b}{a}\right)
$$

The phase angle needs to be derived using a four-quadrant inverse tangent $(\operatorname{atan} 2)$. If the two-quadrant inverse tangent is used, a correction of $\varphi$ for $\pm \pi$ may be necessary as this function is only defined for $-\pi / 2<\varphi<\pi / 2$.

For multi-channel data acquisition with sinusoidal signals, it is advantageous to fit all the channels together with a common frequency [8]. The frequency is not known well enough, so it needs to be estimated as well. The parameters of interest of the three sinusoidal oscillations of the acquired channels $(A, B, C, D, E, G)$ can be estimated with a common excitation frequency $F$ by the function $\operatorname{Multsin}(\boldsymbol{X})$ as follows

$$
\begin{aligned}
\boldsymbol{y} & =\operatorname{Multsin}(\boldsymbol{X}) \\
& =\boldsymbol{x}_{1} \cdot\left(A \cdot \cos \left(2 \pi F \cdot \boldsymbol{x}_{\mathrm{t}}\right)+B \cdot \sin \left(2 \pi F \cdot \boldsymbol{x}_{\mathrm{t}}\right)\right) \\
& +\boldsymbol{x}_{2} \cdot\left(C \cdot \cos \left(2 \pi F \cdot \boldsymbol{x}_{\mathrm{t}}\right)+D \cdot \sin \left(2 \pi F \cdot \boldsymbol{x}_{\mathrm{t}}\right)\right) \\
& +\boldsymbol{x}_{3} \cdot\left(E \cdot \cos \left(2 \pi F \cdot \boldsymbol{x}_{\mathrm{t}}\right)+G \cdot \sin \left(2 \pi F \cdot \boldsymbol{x}_{\mathrm{t}}\right)\right)
\end{aligned}
$$

with the vector of measurement data $\boldsymbol{y}$ and the time and assignment matrix $\boldsymbol{X}$ consisting of the time vector $\boldsymbol{x}_{t}$ and the assignment vectors $\boldsymbol{x}_{1} \ldots \boldsymbol{x}_{3}$ for the different channels, respectively. The length of the vector and the matrix is $3 n$, while $n$ is the number of measurement values acquired.

$$
\begin{aligned}
& \boldsymbol{y}=\left[y_{11}, y_{12}, \ldots, y_{1 n}, y_{21}, y_{22}, \ldots, y_{2 n}, y_{31}, y_{32}, \ldots, y_{3 n}\right]^{T} \\
& \boldsymbol{X}=\left[\boldsymbol{x}_{t} \boldsymbol{x}_{1} \boldsymbol{x}_{2} \boldsymbol{x}_{3}\right]
\end{aligned}
$$

$\boldsymbol{X}=\left[\begin{array}{cccc}x_{11} & 1 & 0 & 0 \\ x_{12} & 1 & 0 & 0 \\ \vdots & 1 & 0 & 0 \\ x_{1 n} & 1 & 0 & 0 \\ x_{21} & 0 & 1 & 0 \\ \vdots & 0 & 1 & 0 \\ x_{2 n} & 0 & 1 & 0 \\ x_{31} & 0 & 0 & 1 \\ \vdots & 0 & 0 & 1 \\ x_{3 n} & 0 & 0 & 1\end{array}\right]$
The unknown parameters $\boldsymbol{\theta}=[A, B, C, D, E, F]$ will be identified by a nonlinear least squares approach as follows

$\widehat{\boldsymbol{\theta}}=\arg \min _{\theta} \sum_{i=1}^{n}(\boldsymbol{y}-\operatorname{Multsin}(\boldsymbol{X}))^{2}$

If the values of the different acquired channels have different numerical magnitudes (due to units, etc.) the least squares algorithm would unintentionally 'weight' the channels according to their numerical magnitudes. To avoid such behaviour, the three channels need to be normalised. A linear least squares regression is applied to each channel based on Eq. (4) with the frequency of excitation assumed to be known. Based on the fit results for each single channel, the three channels are normalised prior to the combined regression described in Eq. (6). The results from the linear regression are used as initial parameters for the iterative combined regression algorithm as described in Eq. (10).

\section{COMPONENTS INFLUENCING THE ACQUIRED SIGNALS}

Prior to the parameter identification, all the dynamic effects of the different components apart from the DUT need to be corrected by means of calibration frequency response functions. This includes the measuring components of the calibration device, as well as the signal conditioning and transmission electronics of the transducer under test. The data acquisition system needs to be calibrated as well.

For each component analysed, a complex frequency response function $H(\mathrm{i} \omega)$ with the output $Y(\mathrm{i} \omega)$ and the input $X(\mathrm{i} \omega)$ was determined for certain calibration frequencies as follows

$$
H(\mathrm{i} \omega)=\frac{Y(\mathrm{i} \omega)}{X(\mathrm{i} \omega)}
$$

The magnitude of the frequency response function follows from

$$
A(\omega)=|H(\mathrm{i} \omega)|=\sqrt{\operatorname{Re}(H(\mathrm{i} \omega))^{2}+\operatorname{Im}(H(\mathrm{i} \omega))^{2}}
$$

with the real part $\mathrm{Re}$ and the imaginary part Im of the frequency response function. The phase $\theta$ of the frequency response function equals

$$
\theta(\omega)=\arctan \left(\frac{\operatorname{Im}(H(\mathrm{i} \omega))}{\operatorname{Re}(H(\mathrm{i} \omega))}\right)
$$

again, as in Eq. 5, it is necessary to use a four-quadrant inverse tangent algorithm to derive the correct phase angle. The acquired measuring signals can now be corrected for magnitude and phase. With the corrected magnitude $A_{\text {corr }}(\omega)$ and phase $\theta_{\text {corr }}(\omega)$, the measured magnitude $A_{\text {meas }}(\omega)$ and phase $\theta_{\text {meas }}(\omega)$ and the magnitude and phase frequency response functions $A_{\text {cal }}(\omega), \theta_{\text {cal }}(\omega)$ from calibration follows

$$
\begin{aligned}
& A_{\text {corr }}(\omega)=A_{\text {meas }}(\omega) \cdot A_{\text {cal }}(\omega)^{-1} \\
& \theta_{\text {corr }}(\omega)=\theta_{\text {meas }}(\omega)-\theta_{\text {cal }}(\omega)
\end{aligned}
$$

\section{PARAMETER IDENTIFICATION}

Mounting different devices under test with different properties (MMOI, torsional stiffness, or damping) will influence the frequency response of the measuring device (see Figure 2). Utilising this variability in the frequency response of 
the measuring device, the properties of the DUT may be identified. The output of the transducer $u_{\text {DUT }}$ is assumed to be proportional to the difference $\Delta_{\mathrm{HB}}$, of the torsion angles at the top $\left(\varphi_{\mathrm{H}}\right)$ and at the bottom $\left(\varphi_{\mathrm{B}}\right)$ of the transducer as

$$
u_{\mathrm{DUT}}(t)=\rho \cdot\left(\varphi_{\mathrm{H}}(t)-\varphi_{\mathrm{B}}(t)\right)=\rho \cdot \Delta_{\mathrm{HB}}(t)
$$

because of the measurement principle (strain gauges) and the known linear behaviour. As the transducer under test measures the torque and not the angle difference, a scale factor $\rho$ is introduced.

For the parameter identification, all the signals acquired are assumed to be harmonic. In this case we can assume the following relationship for the angle $\varphi$, the angular velocity $\dot{\varphi}$ and the angular acceleration $\ddot{\varphi}$ :

$$
\begin{aligned}
& \varphi(t)=\hat{\varphi} \cdot \mathrm{e}^{\mathrm{i} \omega t} \\
& \dot{\varphi}(t)=\mathrm{i} \omega \hat{\varphi} \cdot \mathrm{e}^{\mathrm{i} \omega t}=\mathrm{i} \omega \varphi(t) \\
& \ddot{\varphi}(t)=-\omega^{2} \hat{\varphi} \cdot \mathrm{e}^{\mathrm{i} \omega t}=-\omega^{2} \varphi(t)
\end{aligned}
$$

Here, $\mathrm{i}=\sqrt{-1}$ denotes the imaginary number.

The parameters of the DUT will be identified by analysing the output of the transducer and the mechanical input, which is measured as the angular accelerations $\ddot{\varphi}_{\mathrm{M}}$ and $\ddot{\varphi}_{\mathrm{E}}$ at the top and at the bottom of the coupling elements (see Figure 3). This leads to the following frequency response equations:

$$
\begin{aligned}
& H_{\text {top }}(\mathrm{i} \omega)=\frac{\rho \cdot \Delta_{\mathrm{HB}}}{\ddot{\varphi}_{\mathrm{M}}} \\
& H_{\text {bott }}(\mathrm{i} \omega)=\frac{\rho \cdot \Delta_{\mathrm{HB}}}{\ddot{\varphi}_{\mathrm{E}}}
\end{aligned}
$$

These equations are based on the ordinary differential equation (ODE) system of the model (equations (1), (2a), (2b) (2c), (2d), (2e) (2f), (2g)) and contain the known model parameters of the measuring device, as well as the still unknown parameters of the DUT.

$\Delta_{\mathrm{HB}}=\frac{\omega^{2} J_{\mathrm{M} 2} \varphi_{\mathrm{M}}}{\left(\mathrm{i} \omega d_{\mathrm{T}}+c_{\mathrm{T}}\right)}$

$+\left(\frac{\omega^{2}\left(J_{\mathrm{M} 1}+J_{\mathrm{H}}\right)}{\left(\mathrm{i} \omega d_{\mathrm{T}}+c_{\mathrm{T}}\right)}\left(\frac{-\omega^{2} J_{\mathrm{M} 2}+\mathrm{i} \omega d_{\mathrm{M}}+c_{\mathrm{M}}}{\mathrm{i} \omega d_{\mathrm{M}}+c_{\mathrm{M}}}\right) \varphi_{\mathrm{M}}\right)$

For the transfer function of the top part $H_{\text {top }}(\mathrm{i} \omega)$, there follows

$H_{\text {top }}(\mathrm{i} \omega)=-\rho \cdot \frac{J_{\mathrm{M} 2}+\left(J_{\mathrm{M} 1}+J_{\mathrm{H}}\right) \cdot K_{\mathrm{t}}(\mathrm{i} \omega)}{\mathrm{i} \omega d_{\mathrm{T}}+c_{\mathrm{T}}}$

with

$K_{\mathrm{t}}(\mathrm{i} \omega)=\frac{\varphi_{\mathrm{H}}}{\varphi_{\mathrm{M}}}=\frac{-\omega^{2} J_{\mathrm{M} 2}+\mathrm{i} \omega d_{\mathrm{M}}+c_{\mathrm{M}}}{\mathrm{i} \omega d_{\mathrm{M}}+c_{\mathrm{M}}}$

consisting only of the known model parameters of the measuring device. The expression for $H_{\text {bott }}(i \omega)$ is more complex. Additionally to Eq. (20) we denote

$K_{\mathrm{b}}(\mathrm{i} \omega)=\frac{-\omega^{2} J_{\mathrm{E} 2}+\mathrm{i} \omega d_{\mathrm{E}}+c_{\mathrm{E}}}{\mathrm{i} \omega d_{\mathrm{E}}+c_{\mathrm{E}}}$

which again is not dependent on the DUT's parameters.

With $K_{\mathrm{b}}(\mathrm{i} \omega)$ and $K_{\mathrm{t}}(\mathrm{i} \omega)$ we finally obtain $H_{\text {bott }}(i \omega)$, the transfer function of the bottom part of the measuring device

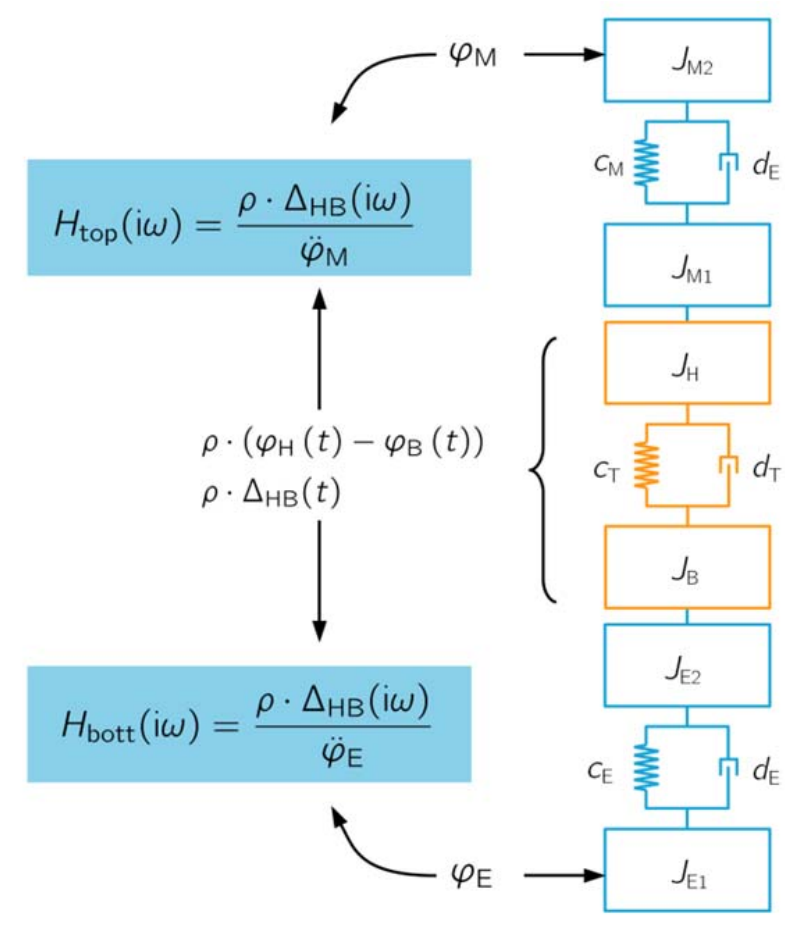

Figure 3. Transfer function based on the acquired measurement channels.

Examining Eq. (22), the simple numerator and complex denominator suggest considering the inverse $H_{\text {bott }}(i \omega)^{-1}$ instead:

$$
\begin{array}{r}
\frac{1}{H_{\mathrm{bott}}(\mathrm{i} \omega)}=\frac{\omega^{2}}{\rho}\left(\frac{-\omega^{2} J_{\mathrm{B}}}{\mathrm{i} \omega d_{\mathrm{E}}+c_{\mathrm{E}}}+\frac{\mathrm{i} \omega d_{\mathrm{T}}+c_{\mathrm{T}}}{\mathrm{i} \omega d_{\mathrm{E}}+c_{\mathrm{E}}}+K_{\mathrm{b}}(\mathrm{i} \omega)\right) \\
+\frac{K_{\mathrm{t}}(\mathrm{i} \omega)}{H_{\mathrm{top}}(\mathrm{i} \omega)}\left(\frac{-\omega^{2} J_{\mathrm{B}}}{\mathrm{i} \omega d_{\mathrm{E}}+c_{\mathrm{E}}}+K_{\mathrm{b}}(\mathrm{i} \omega)\right)
\end{array}
$$

or alternatively from Eq. (19)

$$
\begin{aligned}
& \frac{1}{H_{\mathrm{bott}}(i \omega)}=\frac{\omega^{2}}{\rho}\left(\frac{-\omega^{2} J_{\mathrm{B}}}{\mathrm{i} \omega d_{\mathrm{E}}+c_{\mathrm{E}}}+\frac{\mathrm{i} \omega d_{\mathrm{T}}+c_{\mathrm{T}}}{\mathrm{i} \omega d_{\mathrm{E}}+c_{\mathrm{E}}}+K_{\mathrm{b}}(\mathrm{i} \omega)\right) \\
& -\frac{K_{\mathrm{t}}(\mathrm{i} \omega)}{\rho}\left(\frac{\left(\frac{-\omega^{2} J_{\mathrm{B}}}{\mathrm{i} \omega d_{\mathrm{E}}+c_{\mathrm{E}}}+K_{\mathrm{b}}(\mathrm{i} \omega)\right) \cdot\left(\mathrm{i} \omega d_{\mathrm{T}}+c_{\mathrm{T}}\right)}{J_{\mathrm{M} 2}+\left(J_{\mathrm{M} 1}+J_{\mathrm{H}}\right) \cdot K_{\mathrm{t}}(\mathrm{i} \omega)}\right)
\end{aligned}
$$

which shows the dependencies on the unknown parameters $\rho, c_{\mathrm{T}}, d_{\mathrm{T}}, J_{\mathrm{H}}$ and $J_{\mathrm{B}}$.

To get a closer look into the structure of Eq. (24), we denote

$$
\begin{aligned}
K_{\mathrm{g}}\left(\mathrm{i} \omega, J_{\mathrm{B}}, J_{\mathrm{H}}\right)= & \frac{\omega^{2}}{\mathrm{i} \omega d_{\mathrm{E}}+c_{\mathrm{E}}} \\
& -\frac{K_{\mathrm{t}}(\mathrm{i} \omega) \cdot\left(\frac{-\omega^{2} J_{\mathrm{B}}}{\mathrm{i} \omega d_{\mathrm{E}}+c_{\mathrm{E}}}+K_{\mathrm{b}}(\mathrm{i} \omega)\right)}{J_{\mathrm{M} 2}+\left(J_{\mathrm{M} 1}+J_{\mathrm{H}}\right) \cdot K_{\mathrm{t}}(\mathrm{i} \omega)}
\end{aligned}
$$


Then, using Eq. (25) leads to

$$
\begin{aligned}
\frac{1}{H_{\text {bott }}(i \omega)} & =\frac{\omega^{2}}{\rho} \cdot\left(\frac{-\omega^{2} J_{\mathrm{B}}}{\mathrm{i} \omega d_{\mathrm{E}}+c_{\mathrm{E}}}+K_{\mathrm{b}}(\mathrm{i} \omega)\right) \\
& +\frac{\mathrm{i} \omega d_{\mathrm{T}}}{\rho} \cdot K_{\mathrm{g}}\left(\mathrm{i} \omega, J_{\mathrm{B}}, J_{\mathrm{H}}\right)+\frac{c_{\mathrm{T}}}{\rho} \cdot K_{\mathrm{g}}\left(\mathrm{i} \omega, J_{\mathrm{B}}, J_{\mathrm{H}}\right)
\end{aligned}
$$

expressing the separability of the model for the parameters $1 / \rho, c_{\mathrm{T}} / \rho$ and $d_{\mathrm{T}} / \rho$. These parameters are conditionally linear. That means, when some of the parameters in the equation are known (in this case $J_{\mathrm{B}}, J_{\mathrm{H}}$ ), we deal with a linear model (with respect to the remaining parameters). The same applies to Eq. (19) where $H_{\text {top }}(\mathrm{i} \omega)^{-1}$ gives

$$
\begin{aligned}
\frac{1}{H_{\mathrm{top}}(\mathrm{i} \omega)}= & -\frac{\mathrm{i} \omega d_{\mathrm{T}}}{\rho} \cdot \frac{1}{J_{\mathrm{M} 2}+\left(J_{\mathrm{M} 1}+J_{\mathrm{H}}\right) \cdot K_{\mathrm{t}}(\mathrm{i} \omega)} \\
& -\frac{c_{\mathrm{T}}}{\rho} \cdot \frac{1}{J_{\mathrm{M} 2}+\left(J_{\mathrm{M} 1}+J_{\mathrm{H}}\right) \cdot K_{\mathrm{t}}(\mathrm{i} \omega)}
\end{aligned}
$$

with $c_{\mathrm{T}} / \rho$ and $d_{\mathrm{T}} / \rho$ being conditionally linear.

Assuming that $J_{\mathrm{B}}$ and $J_{\mathrm{H}}$ were known in Eq. (26), or $J_{\mathrm{H}}$ in Eq. (27), additionally to the predetermined parameters of the measuring device, there would be closed-form formulae for the estimation of the remaining parameters $1 / \rho, c_{\mathrm{T}} / \rho$ and $d_{\mathrm{T}} / \rho$ (Eq. (26)) or $c_{\mathrm{T}} / \rho$ and $d_{\mathrm{T}} / \rho$ (Eq. (27)) by means of a linear least squares approach. These formulae would of course depend on $J_{\mathrm{B}}$ and $J_{\mathrm{H}}$ which has implications on the estimation procedure for all the unknown model parameters (see Table 1).

Instead of estimating the 5 parameters of Eq. (26) or the 4 parameters of Eq. (27), respectively, by a least squares approach in one nonlinear optimisation step, the parameters $1 / \rho, c_{\mathrm{T}} / \rho$ and $d_{\mathrm{T}} / \rho$ may be replaced by closed-form formulae, and a nonlinear minimisation over two dimensions $\left(J_{\mathrm{B}}\right.$ and $\left.J_{\mathrm{H}}\right)$ can be carried out [9]. Consecutively, the estimates for $1 / \rho, c_{\mathrm{T}} / \rho$ and $d_{\mathrm{T}} / \rho$ will be obtained from the closed-form formulae, with the estimated values of $J_{\mathrm{B}}$ and $J_{\mathrm{H}}$.

\section{ANALYSIS OF THE SENSITIVITY OF PARAMETER IDENTIFICATION BY SIMULATION}

The expected dynamic behaviour of the measuring device with an installed torque transducer was analysed by simulation.

The simulation was carried out based on the model
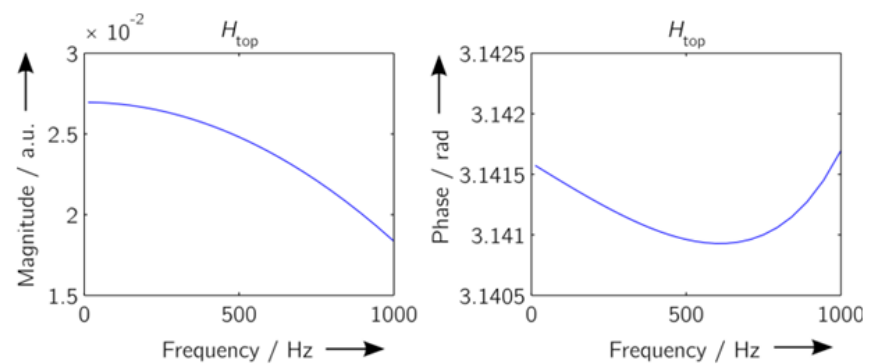

$H_{\text {bott }}$

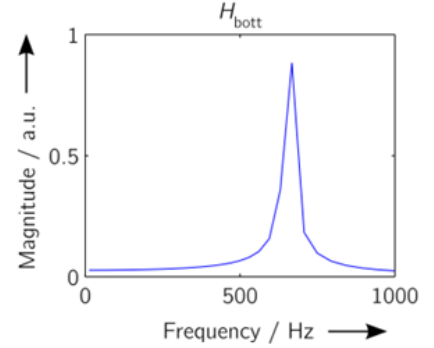

$H_{\text {bott }}$

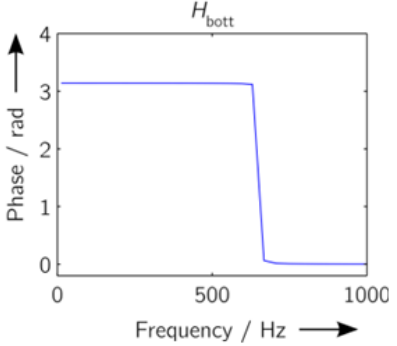

Figure 4. Simulated transfer function $H_{\text {top }}$ (top) and $H_{\text {bott }}$ (bottom) calculated with the parameters of an HBM T10F transducer.
Table 2: Parameters of HBM T5 and HBM T10F transducer from specifications $[10,11]$.

\begin{tabular}{l|ccc} 
& $\begin{array}{c}\text { Torsional stiffness } \\
\left(c_{\mathrm{T}}\right)\end{array}$ & $\begin{array}{c}\text { MMOI } \\
\left(U_{\mathrm{H}}+J_{\mathrm{B}}\right)\end{array}$ & $\begin{array}{c}\text { MMOI } \\
\text { distribution } \\
\left(U_{\mathrm{H}} / J_{\mathrm{B}}\right)\end{array}$ \\
\hline HBM T5 & $640 \mathrm{~N} \cdot \mathrm{m} / \mathrm{rad}$ & $41 \cdot 10^{-6} \mathrm{~kg} \cdot \mathrm{m}^{2}$ & $0.5 / 0.5$ \\
HBM T10F & $160 \cdot 10^{3} \mathrm{~N} \cdot \mathrm{m} / \mathrm{rad}$ & $1.3 \cdot 10^{-3} \mathrm{~kg} \cdot \mathrm{m}^{2}$ & $0.51 / 0.49$
\end{tabular}

equations of the measuring device (Eqs. (1), (2)). The frequency response functions were calculated with chosen parameters for the device under test and with the model parameters of the measuring device. A result of such a simulation is presented in Figure 4, giving the magnitude and phase responses of the complex frequency response functions for the top and for the bottom as depicted in Figure 3.

For the analysis, properties of two typical transducers with a totally different mechanical design - and therefore with different model parameters - were chosen. One transducer is a classical shaft type slip ring transducer of the type HBM T5 (nominal torque $10 \mathrm{~N} \cdot \mathrm{m}$ ) and one is a flange type transducer HBM T10F (nominal torque $50 \mathrm{~N} \cdot \mathrm{m}$ ). Both transducers are used for measurements in the dynamic torque calibration device. The specified model parameters of the analysed transducers are given in Table 2. The MMOI distribution describes to what amount the MMOI of the transducer is allocated in the head $\left(J_{\mathrm{H}}\right)$ and in the base MMOI element $\left(J_{\mathrm{B}}\right)$ of the model.

To find out how well the parameters of transducers under test may be identified, the sensitivity of a change in the parameters of interest $\left(J_{\mathrm{B}}, J_{\mathrm{H}}, c_{\mathrm{T}}, d_{\mathrm{T}}\right.$ and $\left.\rho\right)$ to a change in the theoretical frequency response function (real and imaginary parts) was analysed. Parameters which induce only very small changes in the frequency response function might be difficult to estimate or will have large uncertainties.

Starting from the chosen realistic parameter values, we changed one parameter at a time and compared the resulting frequency response functions with the frequency response functions calculated with the initial set of parameters. This investigation showed that changes in $c_{\mathrm{T}}$ are more pronounced in the real parts of the frequency response functions (see Figure 5), while changes in $d_{\mathrm{T}}$ are manifested more in the imaginary parts. To quantify the induced changes, we
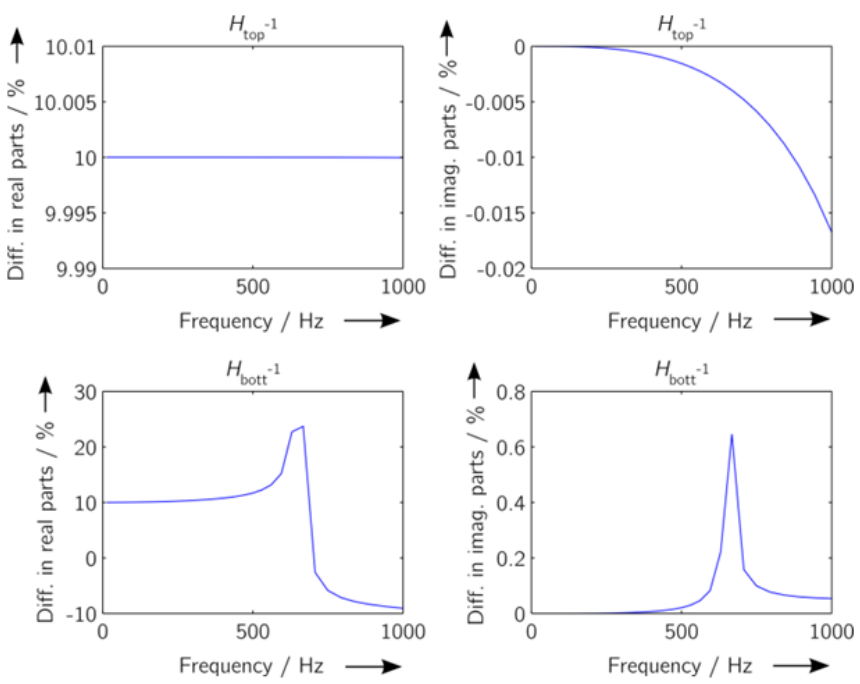

Figure 5. Simulated change of $10 \%$ of $c_{\mathrm{T}}$ of a T10F transducer and its influence on the real and imaginary part of the two frequency response functions function $H_{\text {top }}$ (top) and $H_{\text {bott }}$ (bottom), respectively. 

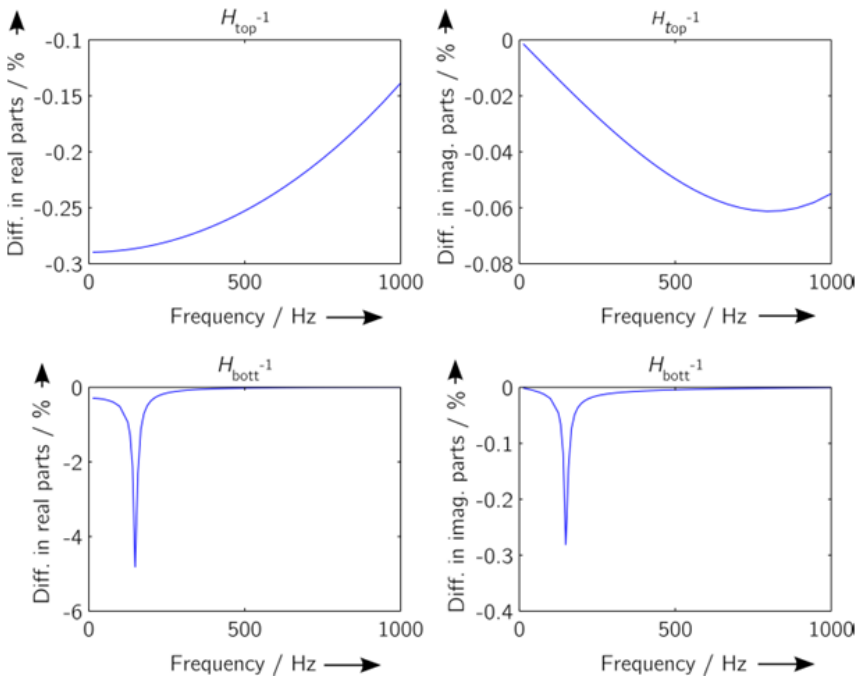

Figure 6. Simulated variation of $10 \%$ of $J_{\mathrm{H}}$ of a T5 transducer and its influence on the real and imaginary parts of the two frequency response functions.
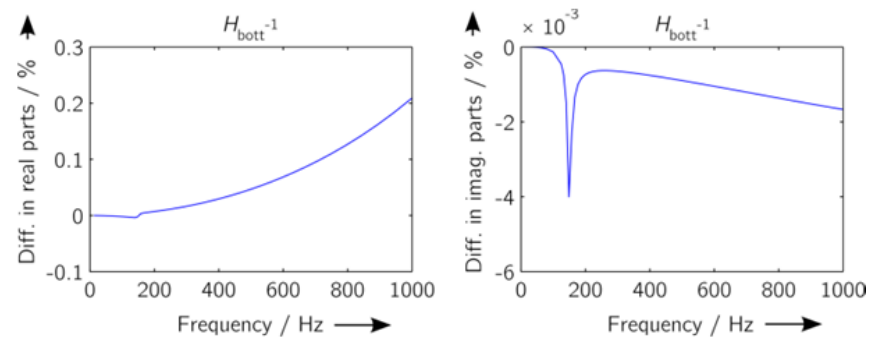

Figure 7. Simulated change of $10 \%$ of $J_{B}$ of a T5 transducer and its only very small influence on the real and imaginary part $H_{\text {bott }}$ (bottom).

considered differences between the real (imaginary) parts as a percentage of the magnitude of the initial inverse frequency response function.

It was found that the mass moments of inertia $J_{\mathrm{B}}$ and $J_{\mathrm{H}}$, of the HBM T5 transducer appear as potentially difficult to identify. A change of $10 \%$ in the value of $J_{\mathrm{H}}$ results in changes of less than $2 \%$ relative to the magnitudes in the real and imaginary parts of this frequency response function aside from the resonance case at about $150 \mathrm{~Hz}$ (see Figure 6). A change of $10 \%$ in the value of $J_{\mathrm{B}}$, which is present only in $H_{\mathrm{bott}}(\mathrm{i} \omega)$, results in changes less than $0.2 \%$ relative to the magnitudes in the real and imaginary parts of this frequency response function, as depicted in Figure 7. Moreover, the influence is more profound for frequencies above the resonance frequency, which are difficult to measure. The same low sensitivity on a variation of a parameter applies for the damping parameter for the HBM T10F transducer.

However, a parameter which is hard to identify means on the other hand that a parameter variation will not influence the dynamic system's behaviour significantly, e.g. the low mass moment of inertia of the HBM T5 transducer (c.f. Table 3) is too small to affect the frequency response of the drive train substantially.

\section{CONCLUSIONS}

The presented identification scheme for the model parameters is a necessary component for the dynamic calibration of torque transducers. The dynamic behaviour of torque transducers is described by a physical model. The model parameters of each transducer will be identified from measurement data acquired during calibration. The model parameters of the measuring device have been determined prior to the calibration measurements to be able to identify the parameters of the transducer's model. For their identification, the angular acceleration at the top and at the bottom of the transducer under test, as well as the transducer's output, will be analysed. Based on this input data, a parameter identification using the method of least squares is presented, and a two stage procedure utilising the separability of the model for a consecutive linear and nonlinear optimisation is described.

Based on the model of the measuring device, an analysis of the influence of the parameter estimation was carried out. It was shown that some transducer parameters might be difficult to identify if they have little influence on the measuring device's dynamic behaviour.

\section{REFERENCES}

[1] C. Bartoli et al., "Traceable Dynamic Measurement of Mechanical Quantities: Objectives and First Results of this European Project" in International Journal of Metrology and Quality Engineering; 3, 127-135 (2012)

DOI: $10.1051 /$ ijmqe/2012020

[2] C. Bartoli et al., "Dynamic Calibration of Force, Torque and Pressure Sensors" in Proc. of Joint IMEKO International TC3, TC5 and TC22 Conference, 2014, Cape Town, South Africa http://www.imeko.org/publications/tc22-2014/IMEKO-TC3TC22-2014-007.pdf

Th. Bruns, "Sinusoidal Torque Calibration: A Design for Traceability in Dynamic Torque Calibration" in Proc. of XVII IMEKO World Congress; 2003, Dubrovnik, Croatia http://www.imeko.org/publications/wc-2003/PWC-2003-TC3008.pdf

[3] L. Klaus, Th. Bruns, M. Kobusch, "Modelling of a Dynamic Torque Calibration Device and Determination of Model Parameters" in ACTA IMEKO Vol. 3, No. 2 (2014), pp. 14-18 http://acta.imeko.org/index.php/acta-

imeko/article/view/IMEKO-ACTA-03\%20\%282014\%29-02$05 / 253$

[4] L. Klaus, B. Arendacká, M. Kobusch, Th. Bruns, "Model Parameter Identification from Measurement Data for Dynamic Torque Calibration" in Proc. of Joint IMEKO International TC3, TC5 and TC22 Conference, 2014, Cape Town, South Africa

http://www.imeko.org/publications/tc3-2014/IMEKO-TC32014-018.pdf

[5] L. Klaus, M. Kobusch, "Experimental Method for the NonContact Measurement of Rotational Damping" in Proc. of Joint IMEKO International TC3, TC5 and TC22 Conference, 2014, Cape Town, South Africa

http://www.imeko.org/publications/tc22-2014/IMEKO-TC3TC22-2014-003.pdf

[6] ISO TC 43. "ISO 266:1997, Acoustics: Preferred frequencies for measurements". International Organization for Standardization (ISO), Geneva, Switzerland, 1997.

[7] P. M. Ramos, A. C. Serra, "A new sine-fitting algorithm for accurate amplitude and phase measurements in two channel acquisition systems" in Measurement Vol. 41, No. 2 (2008), pp. 135-143 DOI: 10.1016/j.measurement.2006.03.011

[8] G. H. Golub, V. Pereyra, "The differentiation of pseudo-inverses and nonlinear least squares problems whose variables separate", SIAM Journal on Numerical Analysis 10, 1973, pp. 413-432. DOI:10.1137/0710036

[9] Hottinger Baldwin Messtechnik GmbH, "T10F Torque Flange Datasheet", 2009.

[10] Hottinger Baldwin Messtechnik GmbH, "T5 Torque Transducer Datasheet", 2004. 